\title{
PARAMETRIC AND NON-PARAMETRIC METHODS FOR THE STUDY OF THE VARIABILITY OF WAVE DIRECTIONS: APPLICATION TO THE ATLANTIC URUGUAYAN COASTS
}

\author{
Sebastián Solari' and Miguel Ángel Losada²
}

\begin{abstract}
Wave direction is a fundamental variable in coastal engineering, whether one is interested in analyzing coastal processes or designing harbor structures. In this work a mixture of circular (wrapped) distributions is introduced for modeling the non-stationary probability distribution function of mean wave directions. The proposed distribution is able to accommodate seasonal and inter-annual variability, as well the influence of several climatic indices. This function was applied to the Atlantic Uruguayan coast, finding that both the Tropical South Atlantic index and the Antarctic Oscillation index have a significant influence on the variability of the wave directions.
\end{abstract}

Keywords: mean wave direction; climatic variability; mixture circular distributions

\section{INTRODUCTION}

To determine the direction and rate of littoral sediment transport, and to analyze the evolution of the coast, the characterization of the seasonal and inter-annual variability in the directional distribution of waves is needed. Moreover, safety and the probability of stoppage of maritime structures under wave action depend on the direction and magnitude of wave action. This variability can be a significant source of uncertainty, affecting decision making in coastal management and the level of operationality of the port and its installations (Solari et al. 2010).

Hemer et al. (2010) studied the variability of wave directions in the southern hemisphere and its connection with large scale climatic process like El Niño/La Niña. This study was based on nonparametric methods (e.g. empirical orthogonal functions (EOF) and linear correlation). Ranasinghe et al. (2004) used historical data to analyze the connection of the wave regimes and the planform of the beach with the Southern Oscillation Index (SOI). However, for engineering applications it is important to have methodologies to quantify and predict the seasonal and inter-annual variability of wave direction distribution, and to thereby provide a tool to narrow the uncertainty in the analysis, design and verification of coastal and port systems.

In this work we: (1) present a parametric non-stationary probability distribution model for wave directions that may be used in the simulation of new time series (see e.g. Solari and Losada 2011, Solari and Losada 2012), (2) identify and quantify the effect of several climatic indices on the seasonal and inter-annual variability of the distribution of wave directions, and include their effect on the parameters of the model, and (3) apply the model to a series of wave directions in the Uruguayan coast.

\section{METHODOLOGY}

We perform two different analyzes on the time series of wave directions and mean monthly anomalies of the climatic indexes: (a) a non-parametric analysis and (b) a parametric analysis, based on the use of a mixture of non-stationary probability distribution functions.

Non-parametric analysis is based on the use of circular moments to identify main variability features of the monthly mean and variance of the data series. The parametric study is methodologically consistent with that proposed by Izaguirre et al. (2011), and allows us to quantify the effect of climate indices on the parameters of the distribution function. The analysis uses a mixture distribution function composed of $N$ Wrapped Normal distributions (WN), as shows in Eq.1

$$
f(\theta)=\sum_{i=1}^{N} \alpha_{i} W N\left(\theta \mid \mu_{i}, p_{i}\right)
$$

where $\Sigma \alpha_{i}=1$ and $0 \leq \alpha_{i} \leq 1$.

The $W N$ distribution function, as seen in the Eq. 2, was developed for circular variables (Fisher 1993).

\footnotetext{
${ }_{1}^{1}$ IMFIA-FING, Universidad de la República, Julio Herrera y Reissig 565, Montevideo, 11300, Uruguay

2 IIISTA-CEAMA, Universidad de Granada, Av. Del Mediterráneo s/n Edif. CEAMA, Granada, 18006, Spain
} 


$$
W N(\theta \mid \mu, p)=\frac{1}{2 \pi}\left(1+2 \sum_{p=1}^{\infty} \rho^{p^{2}} \cos p(\theta-\mu)\right)
$$

with $0 \leq \theta \leq 2 \pi$ and $0 \leq \rho \leq 1$, where $\mu$ is the mean direction and $\rho$ is the mean resultant length that quantifies the dispersion of the distribution. This distribution is thought to be obtained by wrapping a Normal distribution with mean $\mu$ and variance $\sigma^{2}=-2 \log \rho$.

To model the seasonal and inter-annual variations of the distribution, the parameters $\mu_{i}, \rho_{i}$ and $\alpha_{i}$ are expressed as a Fourier series with a main period of one year plus a linear combination of climatic indices. Eq. 3 exemplifies this for the case of the mean

$$
\mu=\mu_{0}+\sum_{k=1}^{n} \mu_{a k} \cos \left(\frac{2 \pi}{k} t\right)+\mu_{b k} \sin \left(\frac{2 \pi}{k} t\right)+\sum \beta_{J} I_{J}(t)
$$

where $t$ is time in years and $I_{J}$ is the monthly anomaly of the climatic indices.

\section{APPLICATION}

\section{Case study description}

A case study was conducted over a period of 21 years (1989-2009) using hindcast mean wave directions (ERA-interim program of the European Centre for Medium-Range Weather Forecasts (ECMWF)) taken at coordinates $36^{\circ} \mathrm{S} 52^{\circ} \mathrm{W}$ (South-American Atlantic coast, see left plot on figure 1).

Given the location of the case study, it is considered that the climatic indices shown in figure 2 may have some influence on the inter-annual variability of the mean wave directions. For each one of these indices, namely Niño 3.4 (NINO34), Tropical South Atlantic Index (TSA), Southern Oscillation Index (SOI) and Antarctic Oscillation (AAO or SAM), the monthly anomaly provided by the National Oceanic and Atmospheric Administration of the US (NOAA Earth System Research Laboratory) was used

A first analysis shows that the distribution of the mean wave directions at the study site is bimodal, with one peak in direction ENE, other in direction S, and the main mass of the data between both (see right plot on figure 1).

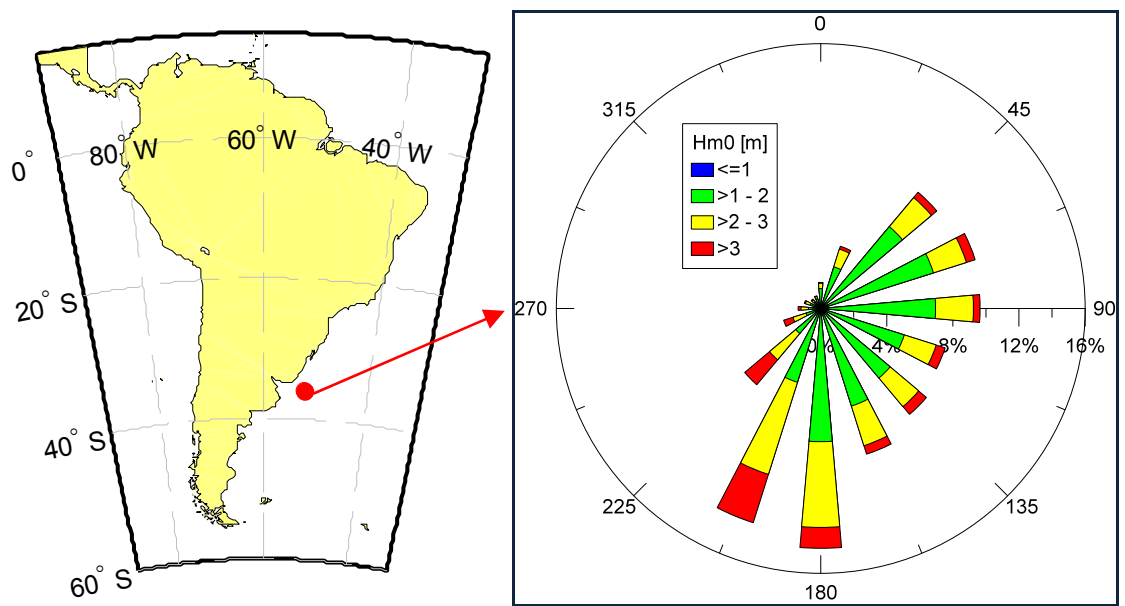

Figure 1. Location of the study site (red dot on left plot) and wave rose (right plot). 


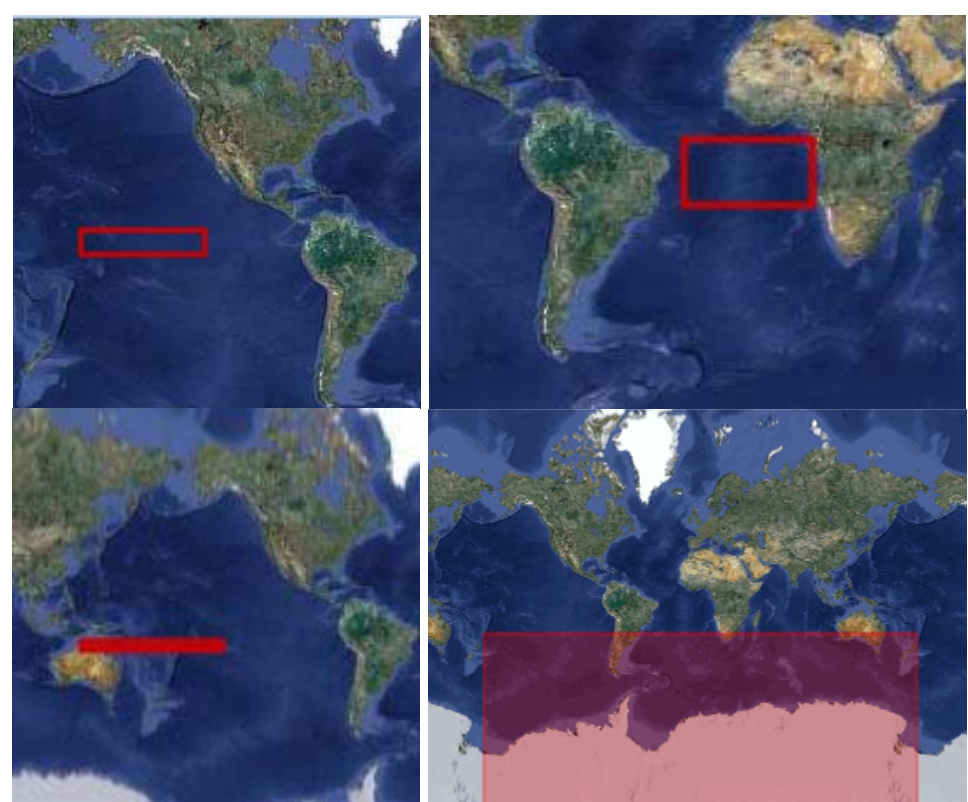

Figure 2. Spatial area considered by the each one of the selected climatic indices. Top-left: Niño 3.4 (NINO34); top-right: Tropical South Atlantic Index (TSA); bottom-left: Southern Oscillation Index (SOI); bottom-right: Antarctic Oscillation (AAO). Images taken from NOAA.
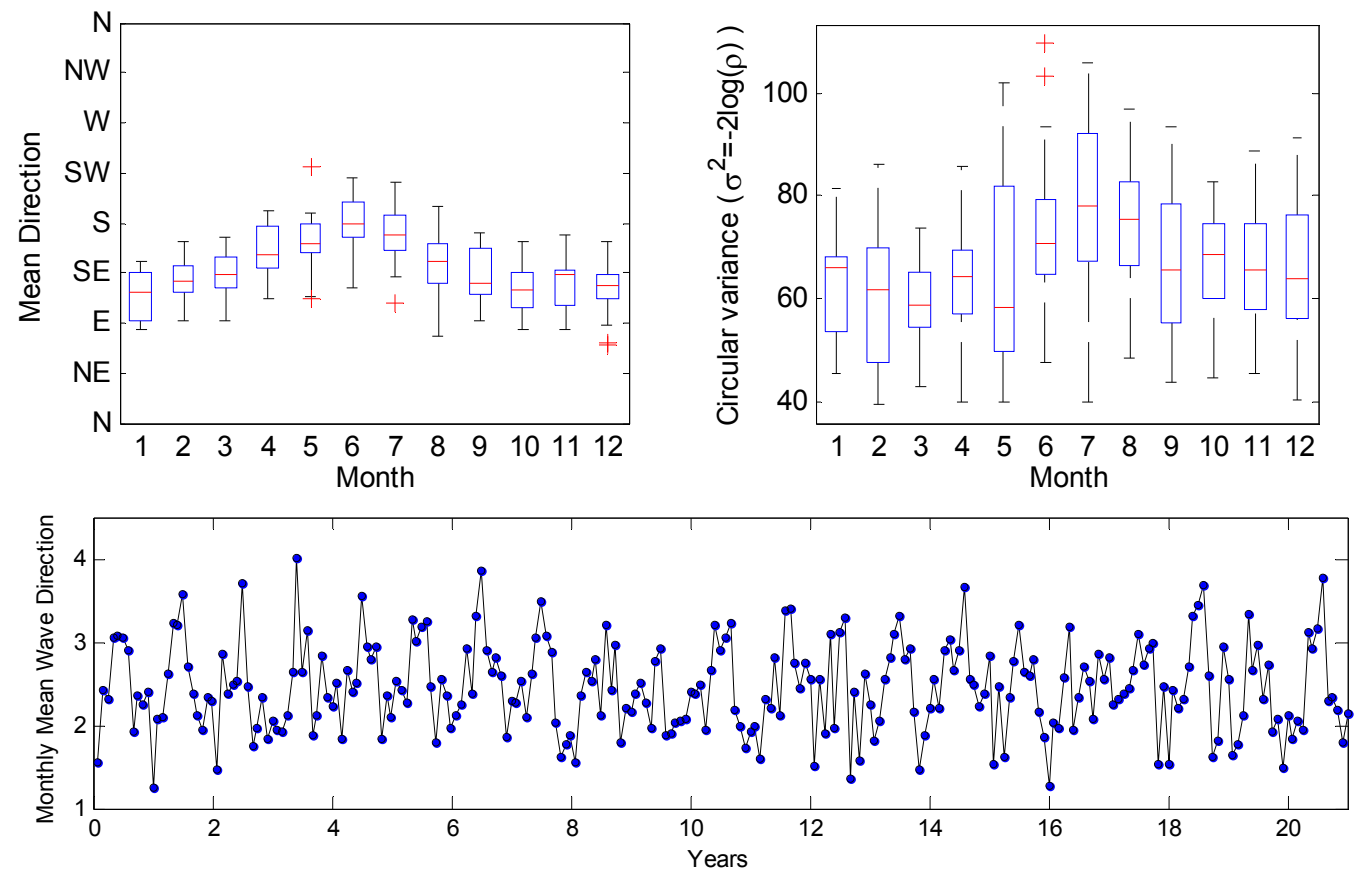

Figure 3. Box plot of monthly means (top-left) and circular variance (top-right), and monthly mean time series (bottom). 


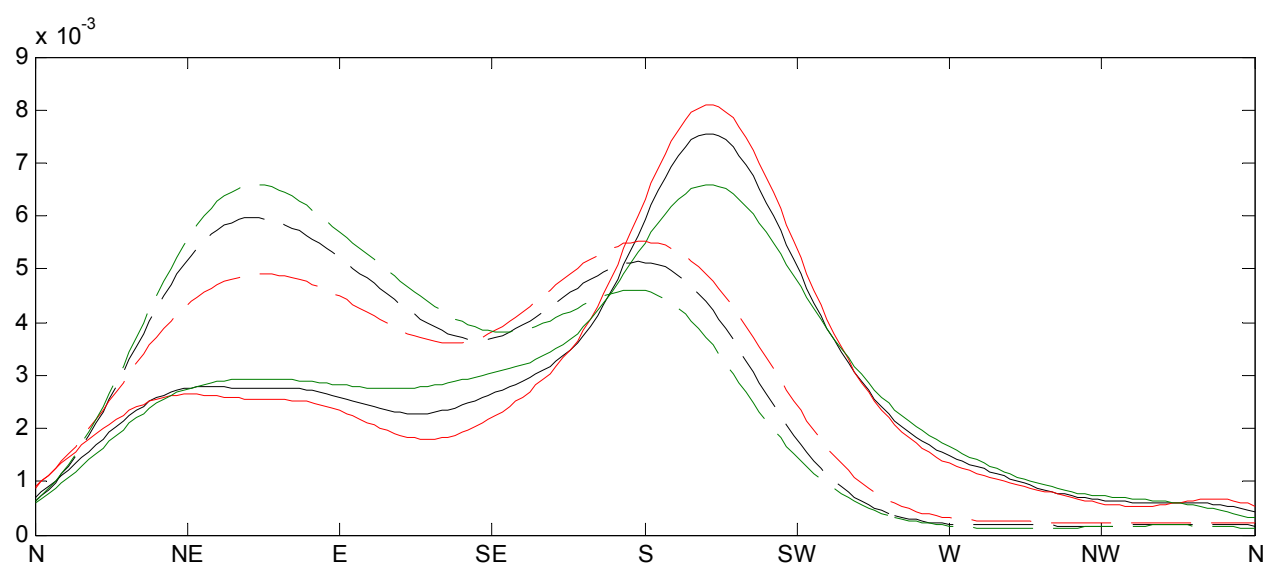

Figure 4. Empirical PDF of the mean wave directions. Continues lines correspond to Julys and dashed lines to Januaries. Black lines corresponds to the PDF estimated with all Januaries (or Julys), red lines correspond to month with $A A O<0$ and green lines to month with $A A O>0$.

\section{Non-parametric analysis}

The use of non-parametric analysis in this work is limited to a preliminary exploratory analysis of the data. Figure 3 (top) shows the box-plots of the monthly mean and variance of the data. Mean and variance were estimated using circular moments (see e.g. Fisher 1993), instead of the most commonly used linear moments. It is noticed that both monthly mean and variance have a significant seasonal cycle, as well as a non-negligible dispersion in the values estimated for any given month. This last point is also noticeable in figure 3 (bottom), where the complete time series of monthly mean wave direction is presented.

From figure 3 it is concluded that both seasonal and inter-annual variability is important in the climate of mean wave directions for our case study. However, when looking at the empirical probability density function in figure 4 , it is notice that an analysis of monthly mean and variance is not enough to characterize the behavior of this variable. We have at least two aspects that are not well represented when analyzing the monthly mean and variance of the data: in first place the PDF is bimodal throughout the year, and mean and variance are unable to provide enough information about the bimodality and may lead to wrong interpretations of the results, and secondly the influence of the climatic indices in the PDF may depends on the range of values of directions that we consider.

\section{Parametric analysis}

Given the results obtained with the non-parametric analysis, it was decided to shift to the use of parametric models for the analysis of the mean wave directions series. For this it was developed a parametric probability distribution function that is able of modeling the observed behavior.

As described in previous section, the distribution developed is a mixture of three wrapped normal distributions $(N=3$ in equation 1$)$, each one having its own mean $(\mu)$, circular variance $(\rho)$ and proportion $(\alpha)$. Seasonal variability within each one of these parameters was modeled by means of a Fourier series approximation of order two $(n=2$ in equation 3$)$, while the possible influence of the indices considered (namely AAO, TSA, SOI and NINO34) was included by means of a linear combination of the monthly anomaly of each index. All the parameters of the model were estimated using the global optimization method Shuffled Complex Evolution, proposed by Duan et al. (1992).

Figure 5 (top left) shows how the proposed model is able to represent the bimodal behavior observed in the mean wave directions distribution. The model fits one WN distribution for each one of the observed modes (one for southern waves and one for nor-eastern waves), and one WN distribution for the gross of the data observed between the two modes.

Figure 5 (top right and bottom left and right) shows the seasonal behavior of the parameters of the mixture distribution. It is seen that: proportion parameters $(\alpha)$ of the $\mathrm{WN}$ distribution corresponding to the modes has a significant seasonal pattern while the one corresponding to the gross of the data is more stable throughout the year; the parameters $\mu$ and $\rho$ of the WN distribution corresponding to the southern mode do not show a seasonal cycle, while the other two distribution do.

With regards to the influence of the climatic indices, figure 6 shows how the mean of each distribution is affected when the monthly anomaly of the indices is included. Although the main variability is imposed by the mean annual cycle, the influence of the indices is not negligible. 
However, to this point there are $32 \beta$ coefficients, four for each parameter of the distribution, so it is desirable to reduce its number in order to facilitate the interpretation of the results. In order to decide which $\beta$ should be kept and which can be assumed to be equal to zero two analyses were performed: firstly a qualitative analysis that provides information on relative influence of $\beta$ when compared with the mean seasonal cycle of the parameters, and secondly a hypothesis test that provides a rigorous methodology to decide which $\beta$ should be discarded.

For the qualitative analysis the $R$ coefficient was defined (equation 4 ). The $R$ coefficient is the ratio between the variation imposed to a given parameter by an anomaly equal to the standard deviation of the whole series of anomalies, and the range of the mean seasonal cycle of the parameter. Table 1 shows the $R$ coefficient for all parameters and indices, and it is noticeable that in many cases the variation produced by some indices is negligible when compared with the seasonal variations, indicating that many $\beta$ may be discarded.

$$
R=\frac{\beta_{I} \sigma_{I}}{\Delta_{\text {SEASONAL }}}
$$

Next, the likelihood ratio test was used in order to define which $\beta$ is significantly different from zero. First, all $\beta$ value whose $95 \%$ confidence interval includes zero are assumed to be equal to zero and are not included in the model. Secondly, all the remaining $\beta$ (those whose $95 \%$ confidence interval do not include zero) are re-estimated.

Table 2 shows the $R$ coefficient calculated with the new $\beta$ parameters. It is noticed that the number of $\beta$ is reduced from 32 to only 9 . However, it should be kept in mind that the final number of $\beta$ values will depend on the value of significance chosen for the analysis (95\% in this case). In table 2 the $\beta$ values with significance greater than $99 \%$ percent are highlighted in bold type, and it is noticed that in this letter case the number of $\beta$ values would had been reduced to only five.
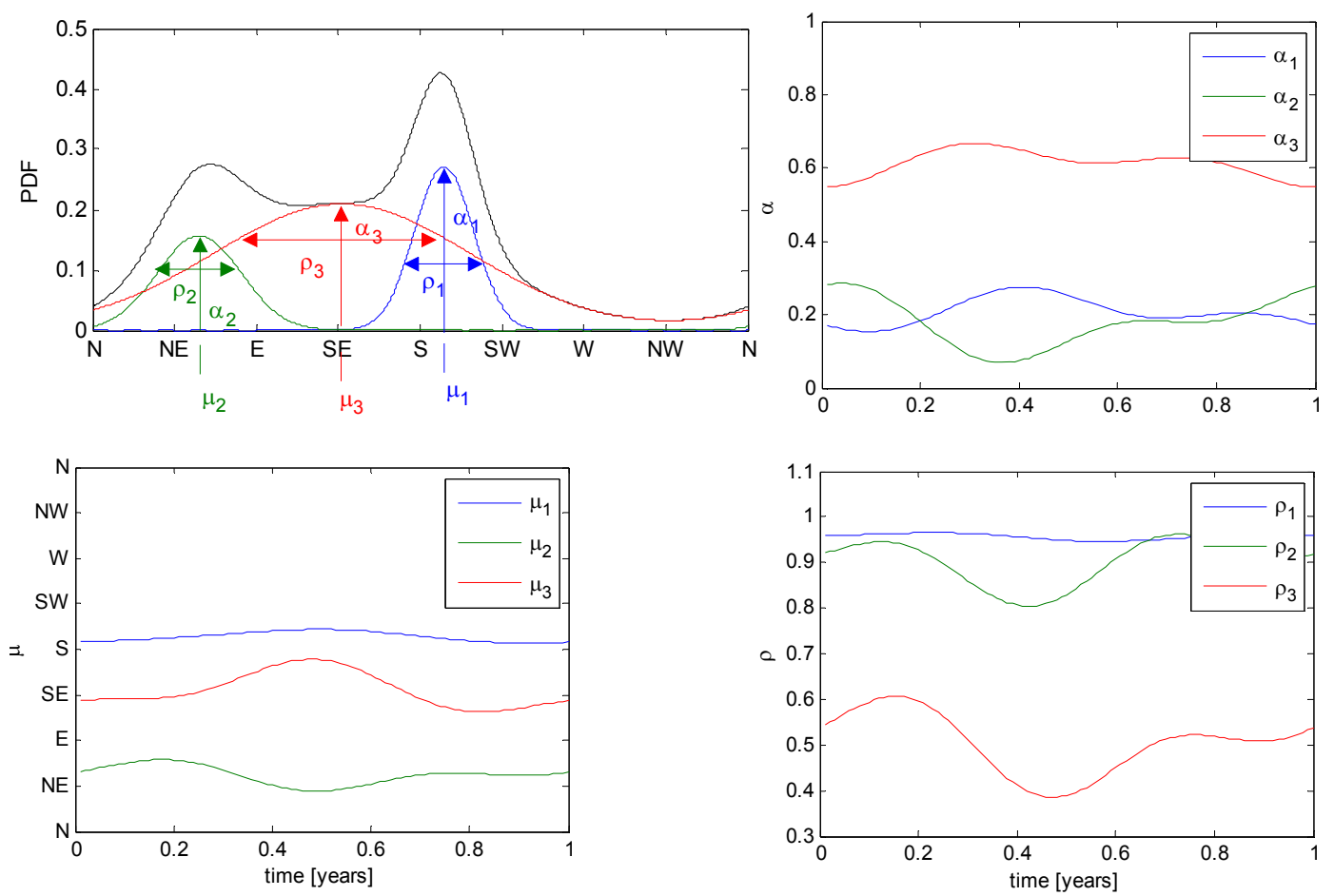

Figure 5. Top left: mean annual stationary PDF of the mixture distribution (black) and the three WN distributions included in the mixture (green, red and blue). Top right: seasonal cycle of proportion parameters ( $\alpha)$. Bottom left and right: seasonal cycle of $\mu$ and $\rho$ parameters. 


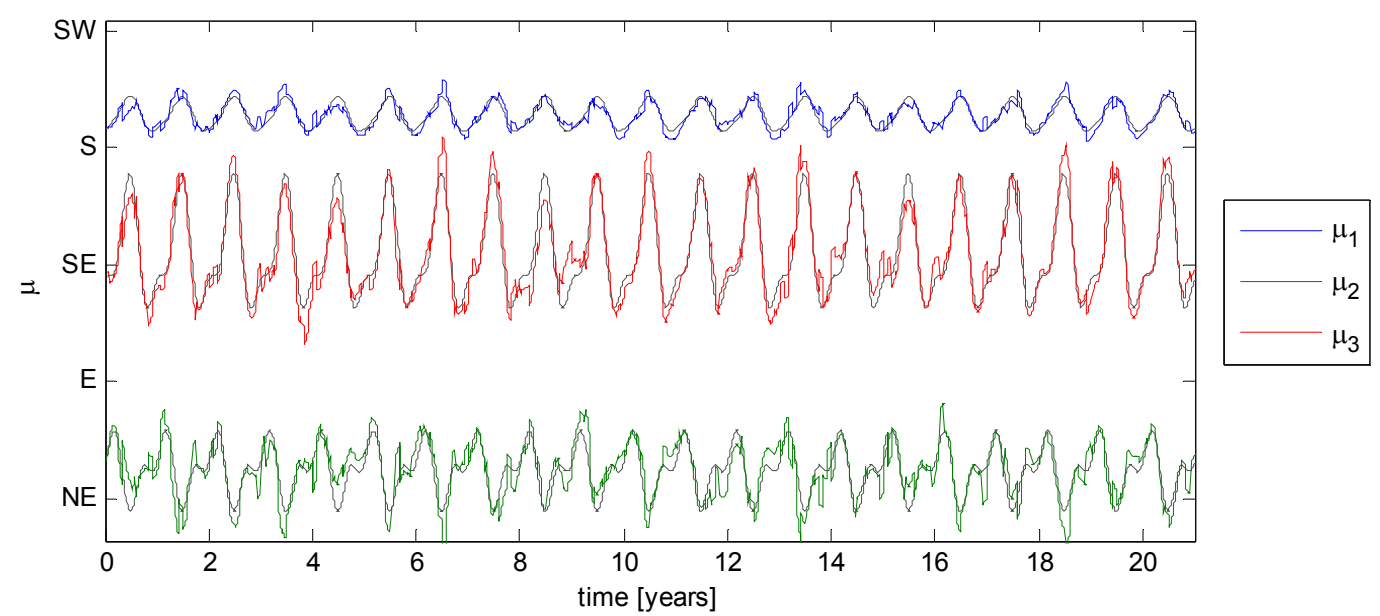

Figure 6. Comparison of the time series of $\mu$ parameters obtained without (grey lines) and with (color lines) the influence of the climatic indices.

\begin{tabular}{|r|r|r|r|r|r|}
\hline \multicolumn{6}{|c|}{$\begin{array}{c}\text { Table 1. Mean value for each parameter and ratio between and } \\
\text { the amplitude of the annual cycle for each climatic index }\left(\mathbf{R}_{\boldsymbol{\beta}}\right) .\end{array}$} \\
\hline Parameter & Mean value & \multicolumn{1}{|c|}{ AAO } & \multicolumn{1}{c|}{ TSA } & SOI & NINO34 \\
\hline $\boldsymbol{\mu}_{1}$ & 193 & $-\mathbf{0 . 1 4}$ & 0.03 & 0.06 & 0.05 \\
\hline $\boldsymbol{\sigma}_{1}{ }^{2}$ & 17 & 0.21 & $\mathbf{0 . 4 2}$ & -0.07 & -0.15 \\
\hline $\boldsymbol{\alpha}_{1}$ & 0.21 & $-\mathbf{0 . 2 4}$ & 0.09 & 0.17 & 0.08 \\
\hline $\boldsymbol{\mu}_{2}$ & 56 & $\mathbf{0 . 1 6}$ & 0.00 & -0.06 & -0.01 \\
\hline $\boldsymbol{\sigma}_{2}{ }^{2}$ & 25 & 0.02 & -0.00 & -0.01 & $-\mathbf{0 . 0 5}$ \\
\hline $\boldsymbol{\alpha}_{2}$ & 0.17 & 0.00 & 0.01 & $-\mathbf{0 . 0 4}$ & 0.01 \\
\hline $\boldsymbol{\mu}_{3}$ & 139 & $-\mathbf{0 . 0 7}$ & 0.06 & -0.04 & -0.01 \\
\hline $\boldsymbol{\sigma}_{3}{ }^{2}$ & 68 & 0.05 & $\mathbf{0 . 0 9}$ & -0.04 & -0.03 \\
\hline
\end{tabular}

\begin{tabular}{|c|c|c|c|c|c|}
\hline Parameter & Mean value & AAO & TSA & sol & NINO34 \\
\hline$\mu_{1}$ & 193 & -0.17 & --- & --- & --- \\
\hline$\sigma_{1}{ }^{2}$ & 17 & --- & 1.46 & --- & -- \\
\hline$a_{1}$ & 0.21 & -0.18 & --- & 0.08 & --- \\
\hline$\mu_{2}$ & 56 & 0.19 & --- & -0.03 & --- \\
\hline$\sigma_{2}{ }^{2}$ & 25 & --- & --- & --- & -- \\
\hline$\alpha_{2}$ & 0.17 & --- & --- & --- & -- \\
\hline$\mu_{3}$ & 139 & -0.09 & 0.24 & --- & --- \\
\hline$\sigma_{3}{ }^{2}$ & 68 & --- & 0.31 & --- & -- \\
\hline
\end{tabular}

The results in table 2 are used to analyze the influence of the climatic indices over the distribution of the wave directions. In this case the indices with the greater influence are Antarctic Oscillation and the Tropical South Atlantic Index. Also, it is noticed that most of the influence is exerted over the WN distribution that represents the southern mode of the distribution (over the waves coming from the south).

Finally, figure 7 shows the superposition of the empirical and modeled annual non-stationary distribution function (PDF). The agreement between the two distributions is noticeable, with the proposed model correctly reproducing the time evolution of the two modes of the empirical distribution.

\section{EXAMPLE APPLICATION}

In order to show the applicability of the proposed model to coastal engineering studies, a simplified example application is presented. 
Here the model is used to estimate how it would be the distribution of the mean wave directions given a set of values of the climatic indices. In particular, the distribution is estimated assuming strong positive and negative anomalies of the Antarctic Oscillation Index.

Figure 8 shows mean annual non-stationary distribution modeled assuming zero AAO anomaly (figure 8 left) and assuming a positive anomaly equal to 1.28 times the standard deviation of the historically observed anomalies (figure 8 right).

Then, the estimated distribution was used to analyze how the sediment transport would be affected by the strong AAO anomaly. For this the rose of annual gross long-shore sand transport was estimated using the CERC formula. Figure 9 shows the roses obtained for the three analyzed cases: zero AAO anomaly and strong positive and negative AAO anomalies. This way an approximation of the morphological variability that can be expected in the Uruguayan coasts may be obtained, even for ungauged conditions.

As said, this is a very simplified example, and it is believed that full potential of the presented model would be exploited when used in probabilistic design, as for example in a Monte Carlo simulation procedure (Losada 2002). An example of the use of non-stationary wrapped distributions for the simulation of wind time series is presented in Mendonça et al. (2012)

\section{CONCLUSIONS}

Conclusions of this work are divided in those related with the model and those related with the case study.

In regard with the model, it was found that mixture of non-stationary circular probability distributions are capable of reproducing the distribution of the mean wave directions, including special features of the distribution like bimodality and dependence on climatic indices. Also, it was found that by retaining only statistically significant parameters, the final number of fitted parameters is reduced and the interpretation of the results is simplified.

In regards with the case study, it was found that Antarctic Oscillation has the greater influence over mean wave directions, followed by the Tropical South Atlantic Index. On the other hand the influence of El Niño/Southern Oscillation is very low.

Future work would investigate whether the influence of the different indices are uniform throughout the year or not, and to develop a simulation methodology for wave time series that makes use of the distribution developed in this work.

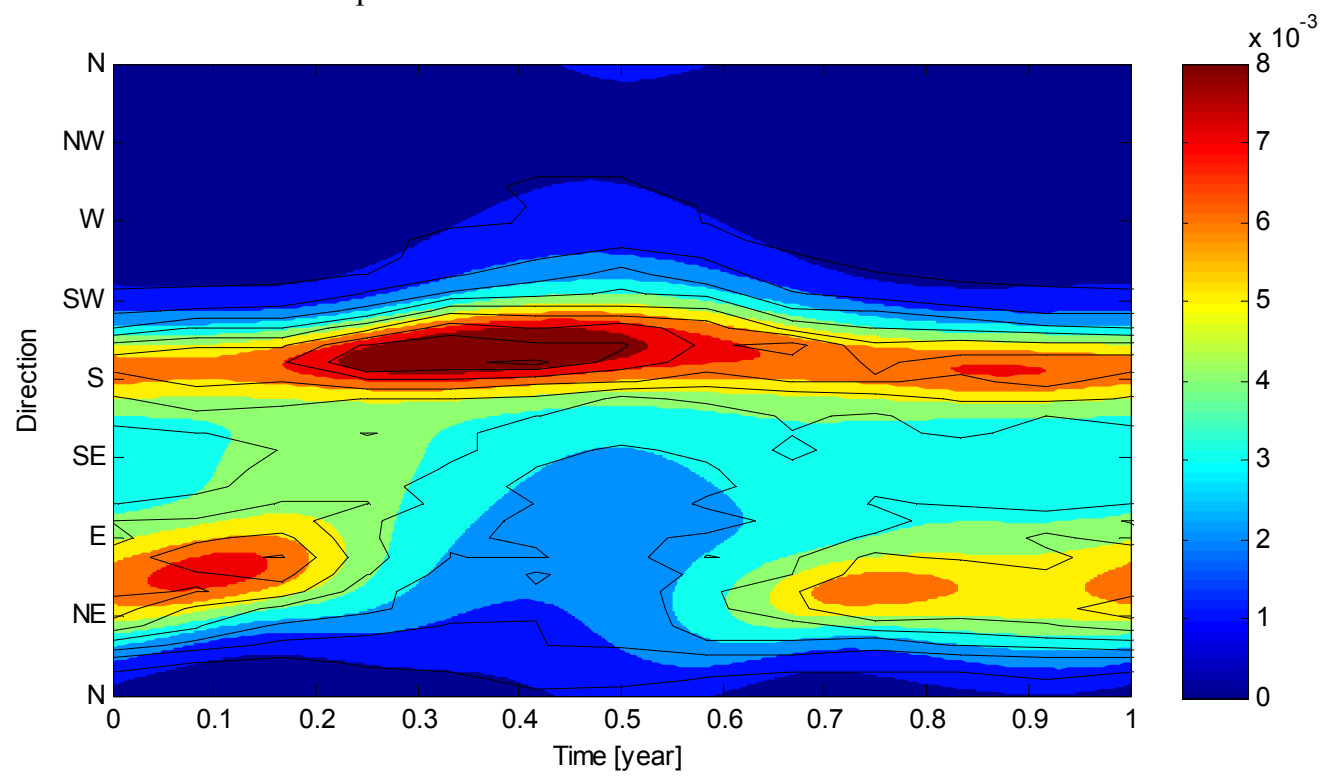

Figure 7. Superposition of the empirical (black lines) and modeled (color filled contours) annual nonstationary distribution function (PDF). 

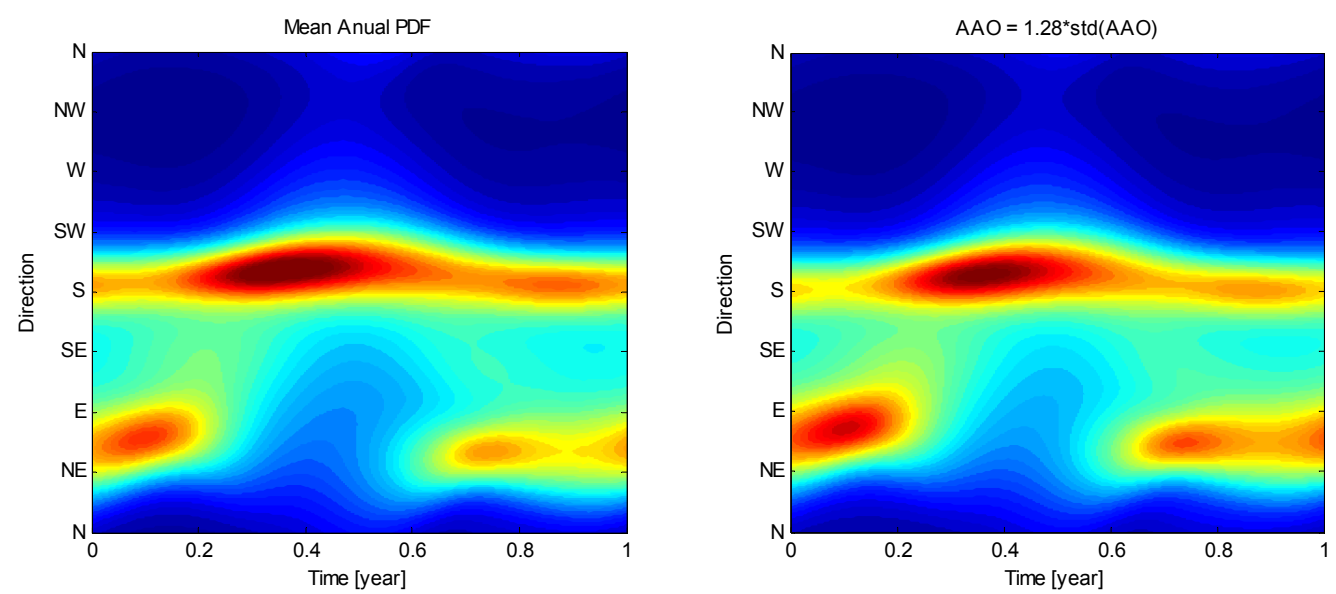

Figure 8. Annual non-stationary distribution function (PDF) considering AAO anomaly equal to zero (left) and equal to 1.28 times the standard deviation of the anomalies (right).
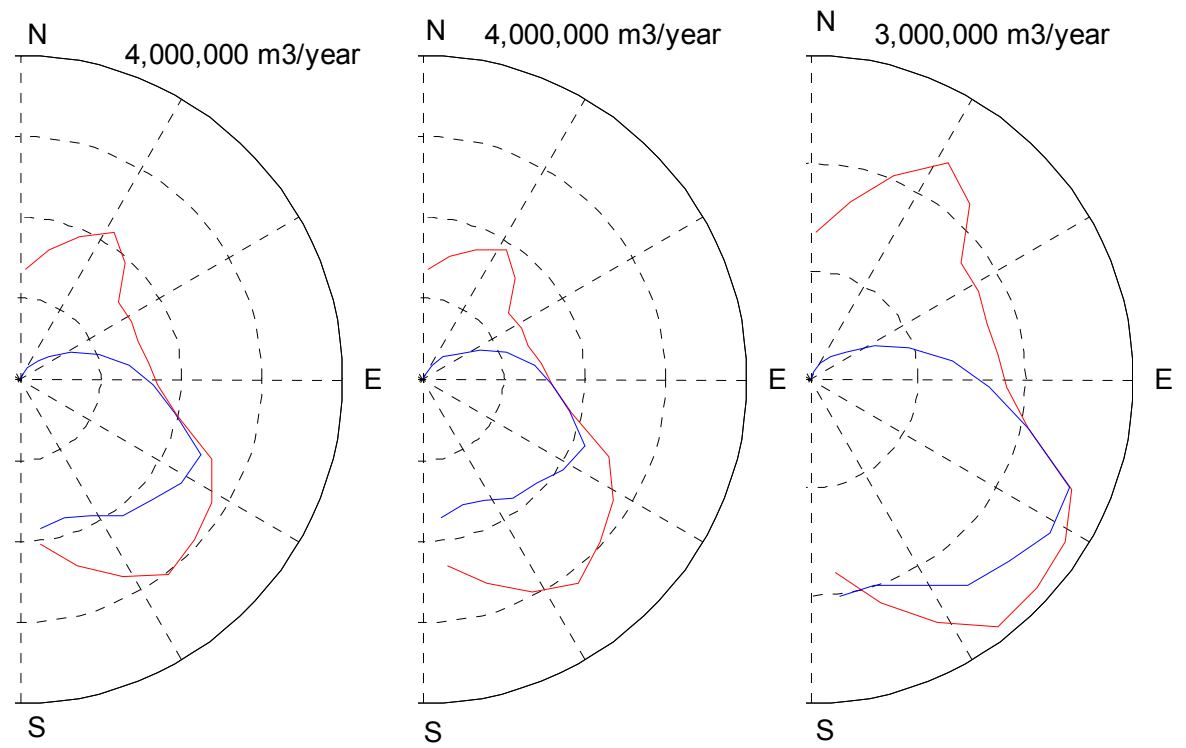

Figure 9. Annual longshore sand transport rates estimated assuming AAO anomaly zero (left), positive (center) and negative (right).

\section{REFERENCES}

Duan, Q., S. Sorooshian, and V. Gupta. 1992. Effective and Efficient Global Optimization for Conceptual Rainfall-Runoff Models, Water Resource Research 28(4) 1015-1031

Fisher, N.I., 1993 Statistical analysis of circular data. Cambridge University Press.

Hemer, A., J.A. Church, and J.R. Hunter. 2010. Variability and trends in the directional wave climate of the Southern Hemisphere, Int. J. Climatol. 30.

Izaguirre, C., F.J. Méndez, M. Menéndez, I.J. Losada. 2011. Global extreme wave height variability based on satellite data, Geophys. Res. Lett. 38.

Losada, M.A., 2002 ROM 0.0 General procedure and requirements in the design of harbor and maritime structures. Part I. Puertos del Estado, editor. ISBN 84-88975-30-9

Mendonça, A., M.A. Losada, S. Solari, M.G. Neves, and M.T. Reis. 2012. Incorporating a risk assessment procedure into submarine outfall projects and application to Portuguese case studies. Proceedings of the $33^{\text {th }}$ International Coastal Engineering Conference.

Ranasinghe, R., R. McLoughlin, A. Short, and G. Symonds. 2004. The Southern Oscillation Index, wave climate, and beach rotation, Marine Geology 204.

Solari, S., A. Moñino, A. Baquerizo, and M.A. Losada. 2010. Simulation model for harbor verification and management. Proceedings of the $32^{\text {nd }}$ International Coastal Engineering Conference. 
Solari, S., and M.A. Losada. 2011. Non-stationary wave height climate modeling and simulation, Journal of Geophysical Research, Vol. 116, C09032, doi:10.1029/2011JC007101.

Solari, S., and M.A. Losada. 2012. Unified distribution models for met-ocean variables: Application to series of significant wave height, Coastal Engineering, Vol. 68, 67-77, doi:10.1016/j.coastaleng.2012.05.004. 